\title{
Risk factors of type 1 gastric neuroendocrine neoplasia in patients with chronic atrophic gastritis. A retrospective, multicentre study
}

\author{
Davide Campana $\mathbb{D}^{1} \cdot$ Davide Ravizza $^{2} \cdot$ Piero Ferolla $^{3}$ - Antongiulio Faggiano ${ }^{4}$. \\ Franco Grimaldi ${ }^{5}$. Manuela Albertelli ${ }^{6,7}$. Claudio Ricci ${ }^{1}$ - Donatella Santini ${ }^{8}$. \\ Nicole Brighi ${ }^{9} \cdot$ Nicola Fazio $^{2} \cdot$ Annamaria Colao $^{4} \cdot$ Diego Ferone $^{6,7}$. \\ Paola Tomassetti ${ }^{1}$
}

Received: 2 May 2016 / Accepted: 18 August 2016 / Published online: 3 September 2016

(C) Springer Science+Business Media New York 2016

\begin{abstract}
The aim of this retrospective study was to evaluate the presence of risk factors for a type 1 gastric neuroendocrine neoplasia in a large cohort of patients with chronic atrophic gastritis. The study design consisted of an Italian multicentre, retrospective analysis. The study included all consecutive patients with chronic atrophic gastritis with or without type 1 gastric neuroendocrine neoplasias followed at the participating centres. Two hundred and twenty-nine patients with chronic atrophic gastritis were enroled at the participating centres. A total of 207 patients (154 female, 53 males, median age: 56.0 years) were included in the final analysis. One hundred and twenty-six
\end{abstract}

Davide Campana

davide.campana@unibo.it

1 Department of Medical and Surgical Sciences, S.Orsola-Malpighi University Hospital, Bologna, Italy

2 Division of Endoscopy and Unit of Gastrointestinal and Neuroendocrine Tumours, European Institute of Oncology, Milan, Italy

3 Department of Medical Oncology, Multidisciplinary NET Centre, Umbria Regional Cancer Network, Umbria, Italy

4 Division of Endocrinology, Department of Clinical Medicine and Surgery, "Federico II" University of Naples, Naples, Italy

5 Endocrinology and Metabolism Unit, University Hospital S. Maria della Misericordia, Udine, Italy

6 Endocrinology, Department of Internal Medicine and Medical Specialties (DiMI) and Centre of Excellence for Biomedical Research (CEBR), University of Genova, Genoa, Italy

7 IRCCS-AOU San Martino-IST, Genova, Italy

8 Dipartimento della medicina diagnostica e della prevenzione, S.Orsola-Malpighi University Hospital, Bologna, Italy

9 Department of Experimental, Diagnostic and Specialty medicine S.Orsola-Malpighi University Hospital, Bologna, Italy patients had chronic atrophic gastritis without a gastric neuroendocrine neoplasia and 81 had a chronic atrophic gastritis with type 1 gastric neuroendocrine neoplasia. The median Chromogranin A level, evaluated in 141 patients, was $52.0 \mathrm{U} / \mathrm{L}$. At upper gastrointestinal endoscopy, atrophy of the gastric mucosa was mild/moderate in 137 patients and severe in 68. Intestinal metaplasia of the corpus was present in 168 patients. At histological examination, 81 patients had a gastric neuroendocrine neoplasia (42 patients had a NET G1 and 33 a NET G2). The median Ki67 index was $2.0 \%$. At univariate and multivariate analysis, the risk factors for a gastric neuroendocrine neoplasia were: male gender, chromogranin A greater than $61 \mathrm{U} / \mathrm{L}$, presence of intestinal metaplasia and age equal to or greater than 59 years. Chromogranin A greater than $61 \mathrm{U} / \mathrm{L}$, the presence of intestinal metaplasia and male gender were independent risk factors for a type 1 gastric neuroendocrine neoplasia in patients with chronic atrophic gastritis.

Keywords Neuroendocrine tumours - Chronic atrophic gastritis · Gastric carcinoid · Gastrin · Chromogranin A

\section{Introduction}

Chronic atrophic gastritis (CAG) is an inflammatory condition characterised by the loss of gastric glandular structures which are replaced by connective tissue (nonmetaplastic atrophy) or by glandular structures inappropriate for location (metaplastic atrophy) [1]. Epidemiological data suggest that CAG is associated with two different types of tumours: intestinal-type gastric cancer and type 1 gastric neuroendocrine neoplasia (Type 1 gNEN). Gastric neuroendocrine neoplasia is derived from enterochromaffin-like cells (ECL-cells) localised in the gastric mucosa [2]. 
Three subtypes of ECL-cell tumours have been recognised: type 1 lesions (70-80\%) are associated with CAG and hypergastrinemia; type 2 lesions $(5-8 \%)$ are associated with gastrinomas in Zollinger-Ellison syndrome and multiple endocrine neoplasia type 1 (MEN 1) and type 3 (15-20\%) are sporadic lesions arising in otherwise normal gastric mucosa without hypergastrinemia [3].

Types 1 gNENs are always associated with an elevated concentration of circulating serum gastrin [3] which exerts a trophic effect on the ECL-cells, leading to hyperplasia and, in some cases, to a true neuroendocrine neoplasm [4]. The majority of type 1 gNENs occur in women and are rarely symptomatic [5]. They are non-functioning tumours, typically found during upper gastrointestinal (GI) endoscopy for dyspepsia or anaemia [6]. Type $1 \mathrm{gNENs}$ frequently present as multiple polyps, usually $<1 \mathrm{~cm}$ in diameter, localised in the gastric corpus-fundus. They are almost exclusively benign lesions with a low risk of deep invasion of the gastric wall [7]. These tumours have a good prognosis with the 5-year survival rate quoted at $96 \%$ which does not differ from an age-matched normal population [5, 8].

The aim of this retrospective study was to evaluate the presence of risk factors for Type $1 \mathrm{gNEN}$ in a large cohort of patients with CAG.

\section{Patients and methods}

\section{Study design and patients}

The study design consisted of an Italian multicentre, retrospective analysis. The study included all consecutive patients with CAG with or without type $1 \mathrm{gNENs}$ followed at Neuroendocrine Tumors Centre of Bologna and patients with CAG with type $1 \mathrm{gNEN}$ followed at the other participating centres (i.e., Milan-IEO, Perugia, Naples, Udine, Genova) from June 1998 to December 2013. Demographic data, as well as clinical and pathological features were collected in a dedicated computerised database and included baseline situations with demographic data, past medical history, symptoms at the diagnosis of the gNEN, evaluations of anti-parietal cell antibodies (APCAs), serum gastrin levels and plasma levels of Chromogranin A ( $\mathrm{CgA})$, findings at endoscopy and at pathological examination (grade of CAG and the presence of intestinal metaplasia).

The inclusion criteria were a histologically confirmed diagnosis of CAG with or without gNENs, and the presence a gastrin level greater than the normal range at diagnosis.

\section{Data analysis}

A dedicated computerised database was created, and demographic data, as well as pathological features, were retrospectively analysed. The histological specimens were examined by an expert NEN-dedicated pathologist at each centre. When required, additional centralised revision of the tumour specimens was carried out. The degree of atrophy of the gastric mucosa was classified according to the updated Sydney system classification [9]. The tumours were classified according to the $2010 \mathrm{WHO}$ classification [10] and the tumour node metastasis classification/G grading system [11]. The Ki67 proliferation index was expressed as a percentage based on the number of Ki67-positive cells in 2000 tumour cells in the areas of the highest immunostaining using the MIB1 antibody (DBA, Milan, Italy).

Median, interquartile ranges (IQRs, 25th to 75th percentiles), ranges and frequencies were used to describe the data. Analysis of the predictive risk factors for neoplasia was carried out by univariate and multivariate analyses using logistic regression. Risk factors were expressed as odds ratio (OR) [95\% confidence interval (CI)]. The multivariate model was constructed using the forward stepwise method after including all variables. All analyses carried out for risk factors are listed in the Tables. The area under (AUC) the receiver-operatingcharacteristic curve (ROC), was evaluated to determine the accuracy of $\mathrm{CgA}$ and age at diagnosis in predicting of neoplasia presence. The best cutoff value was estimated by a maximum likelihood ratio method. Sensitivity and specificity were calculated using the standard formulae (sensitivity $=$ true positive/true positive + false negative and specificity $=$ true negative/true negative + false positive).

Two-tailed $P$ values less than 0.05 were considered statistically significant. The statistical analysis was carried out using dedicated software (IBM-SPSS Statistics version 19.0).

\section{Results}

\section{Study population}

Two hundred and twenty-nine patients with CAG were enroled at the participating centres. Of these, 22 patients $(9.6 \%)$ were excluded because the gastrin level was not available. Thus, a total of 207 patients were included in the final analysis. The characteristics of all 207 patients are listed in Table 1.

There were 154 females $(71.4 \%)$ and 53 males $(25.6 \%)$ with a median age of 56.0 years (range $20-88$ years). One hundred and twenty-six patients $(60.9 \%)$ had CAG without gNENs and 81 (39.1\%) had CAG with type 1 gNENs. One hundred and seventy-four patients $(84.1 \%)$ had autoimmune disease with positive APCAs and $21(10.1 \%)$ were APCAs negative (APCAs not evaluated in 12 patients). 
Table 1 Clinical, biochemical and histological features of 207 CAG patients with or without type 1 gNEN

\begin{tabular}{lccc}
\hline & All patients $n=207$ & Type 1 gNEN $n=81$ & CAG $n=126$ \\
\hline Age, years (IQR) & $56(44-65)$ & $60(46-66)$ & $53(42-64)$ \\
Male gender, $n(\%)$ & $53(25.6)$ & $32(39.5)$ & $21(16.7)$ \\
Gastrin, pg/ml, median (IQR) & $986.0(701.0-1619.0)$ & $1000.0(652.0-1921.0)$ & $977.0(710.0-1562.0)$ \\
Chromogranin A, U/L, median (IQR) & $52.0(33.0-103.0)$ & $83.0(40.0-204.0)$ & $44.0(32.0-77.0)$ \\
PCA, $n(\%)$ & $174(84.1)$ & $63(77.8)$ & $111(88.1)$ \\
Dyspepsia, $n .(\%)$ & $79(38.2)$ & $29(35.8)$ & $50(39.7)$ \\
Intestinal Metaplasia, $n(\%)$ & $168(81.2)$ & $72(88.9)$ & $96(76.2)$ \\
Body-Fundus atrophy, $n(\%)$ & & & $92(73.0)$ \\
$\quad$ Mild/moderate & $137(66.2)$ & $45(55.5)$ & $34(42.0)$ \\
$\quad$ Severe & $68(32.8)$ & $2(2.5)$ & 0 \\
Not evaluated & $2(1.0)$ & $34.0)$ \\
\hline
\end{tabular}

$P C A$ parietal cell antibodies, $C A G$ chronic atrophic gastritis, IQR interquartile ranges

Fig. 1 a Receiver-operatingcharacteristic curve of $\mathrm{CgA}$ in predicting patients who had type 1 gastric neuroendocrine neoplasia. Sensitivity and specificity obtained using the best cutoff values were $61.5 \%$ and $68.5 \%$, respectively. $\mathbf{b}$ Receiver-operating-characteristic curve of age in predicting patients who had type 1 gNEN. Sensitivity and specificity obtained using best cutoff were $55.6 \%$ and $65.1 \%$, respectively. AUC: area under the curve
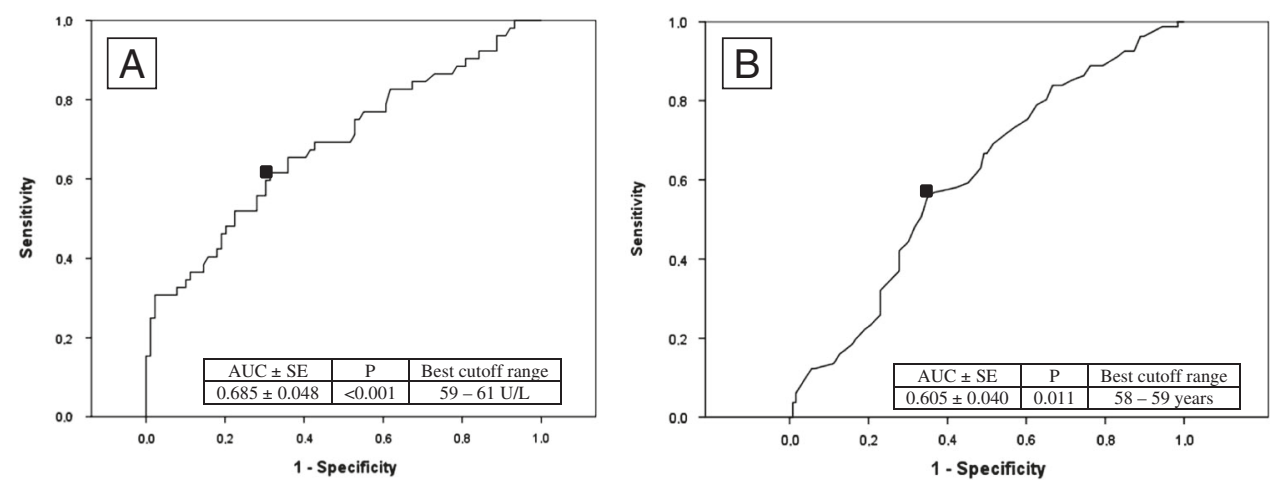

All patients had serum gastrin levels above the normal value (median: $986.0 \mathrm{pg} / \mathrm{ml}$; IQR: 701.0-1619.0 pg/ml). The median CgA level, evaluated in 141 patients $(68.1 \%)$, was 52.0 U/L (IQR: 33.0-103.0 U/L). No patient had a renal insufficiency superior to grade 1 or took proton pump inhibitors at the time of $\mathrm{CgA}$ evaluation. Seventy-nine patients $(38.2 \%)$ had dyspepsia at diagnosis.

At upper gastrointestinal endoscopy and histopathological examination, atrophy of the gastric mucosa was mild/ moderate in 137 patients $(66.2 \%)$, severe in $68(32.8 \%)$ and, in 2 patients $(1.0 \%)$, CAG without grade of atrophy was described. Intestinal metaplasia of the corpus was present in 168 patients $(81.2 \%)$. Ten patients $(4.8 \%)$ had Helicobacter pylori infection at the moment of endoscopic examination. At histological examination, 81(39.1\%) patients (32/53 males and 49/154 females) had neuroendocrine tumours. According to the 2010 WHO classification, 42 patients $(20.3 \%)$ had a NET G1, $33(15.9 \%)$ a NET G2 and 6 patients $(2.9 \%)$ had a NET without evaluation of Ki67 due to the scarcity of the tissue samples. The median Ki67 index was 2.0\% (IQR 1.0-3.6\%). At diagnosis, 71/81 patients $(87.6 \%)$ were in stages $0-\mathrm{I}, 6 / 81(7.4 \%)$ in stage IIA and $4 / 81(5.0 \%)$ in stage IIIB.

In 118/126 patients without gNENs, histological examination showed endocrine cell hyperplasia: linear in $19(16.1 \%)$, micronodular in $93(78.8 \%)$ and adenomatoid in $6(5.0 \%)$. In 7 patients, endocrine cell hyperplasia was absent while in one case no data was available.

\section{Receiver-operating-characteristic curves}

In order to identify $\mathrm{CgA}$ and age cutoff values which could distinguish patients with gNENs from those without, a ROC analysis was carried out.

The ROC curve of the CgA in predicting patients who had gNENs is shown in Fig. 1a. The CgA was accurate (area under the curve \pm SE: $0.685 \pm 0.048 ; P<0.001$ ), and the best cut-off ranged from 59.0 to $61.0 \mathrm{U} / \mathrm{L}$. The sensitivity and specificity obtained using the best cutoff values were $61.5 \%$ and $68.5 \%$, respectively.

The ROC curve of the age in predicting patients who had gNENs is shown in Fig. 1b. The AUC of the age was quite 
Table 2 Risk factors for type 1 gNEN at univariate and multivariate analyses

\begin{tabular}{|c|c|c|c|c|c|c|}
\hline \multirow[t]{2}{*}{ Variable } & \multicolumn{3}{|l|}{ Univariate } & \multicolumn{3}{|l|}{ Multivariate } \\
\hline & Odds ratio & $95 \% \mathrm{CI}$ & $P$ & Odds ratio & $95 \% \mathrm{CI}$ & $P$ \\
\hline Age $>59$ years & 2.33 & $1.32-4.12$ & 0.004 & 2.39 & $1.08-5.27$ & 0.031 \\
\hline Male gender & 3.26 & $1.71-6.23$ & $<0.001$ & 2.59 & $1.09-6.18$ & 0.031 \\
\hline Presence of intestinal metaplasia & 2.50 & $1.12-5.59$ & 0.026 & 4.15 & $1.21-14.21$ & 0.024 \\
\hline Negative APCA & 2.86 & $1.13-7.28$ & 0.027 & - & - & Ns \\
\hline Dyspepsia & 0.85 & $0.48-1.51$ & 0.575 & - & - & Ns \\
\hline Severe CAG & 2.04 & $1.13-3.70$ & 0.018 & - & - & Ns \\
\hline Gastrin* & - & - & 0.281 & - & - & Ns \\
\hline Chromogranin $\mathrm{A} \geq 61 \mathrm{U} / \mathrm{L}$ & 3.27 & $1.62-6.59$ & 0.001 & 4.49 & $2.00-10.06$ & $<0.001$ \\
\hline
\end{tabular}

$A P C A$ anti-parietal cell antibodies, $C A G$ chronic atrophic gastritis, $C I$ confidence interval

* Continuous variable accurate (area under the curve \pm SE: $0.605 \pm 0.040$; $P=0.011$ ), and the best cutoff ranged from 58.0 to 59.0 years. The sensitivity and specificity obtained using the best cutoff values were $55.6 \%$ and $65.1 \%$, respectively.

\section{Risk factors of type 1 gNENs}

At univariate analysis, the variables considered as risk factors for gNENs are summarised in Table 2. The major risk factors for gNENs were male gender (OR: 3.26, $p<$ 0.001 ) and Chromogranin A equal to or greater than $61 \mathrm{U} / \mathrm{L}$ (OR: $3.27, p=0.001$ ). The presence of intestinal metaplasia (OR: $2.50, p=0.026$ ), negative APCA (OR: $2.86, p=$ 0.027), severe CAG (OR: $2.04, p=0.018$ ) and age equal to or greater than 59 years (OR: $2.33, p=0.004)$ represent the other risk factors for gNENs. Circulating levels of gastrin $(p=0.281)$ and the presence of dyspepsia $(p=0.575)$ were not related to the presence of tumour.

$\mathrm{CgA}$ equal to or greater than $61 \mathrm{U} / \mathrm{L}$ (OR: $4.49, p<$ 0.001 ), the presence of intestinal metaplasia (OR: $4.15, p=$ 0.024 ), male gender (OR: $2.59, p=0.031$ ) and age equal to or greater than 59 years (OR 2.39, $p=0.031$ ) were also confirmed at multivariate analysis (Table 2).

\section{Discussion}

In the present study, two large cohorts of patients with CAG were compared, one with gNEN and the other without gNEN, to evaluate the risk factors of gastric neuroendocrine tumours type 1. Using univariate and multivariate analyses, it was found that higher levels of $\mathrm{CgA}$ were associated with the presence of gNEN. The role of $\mathrm{CgA}$ in neuroendocrine tumours is well known $[12,13]$. A recent review has confirmed that $\mathrm{CgA}$ is the most sensitive biomarker in neuroendocrine tumours and is more frequently elevated in well-differentiated tumours as compared to poorly differentiated tumours [14]. Patients with CAG present high values of $\mathrm{CgA}$ [15] due to the trophic action of gastrin on the endocrine cells of the gastric mucosa, which leads to an increase in $\mathrm{CgA}$ levels. Previous studies have shown higher levels of $\mathrm{CgA}$ in patients with $\mathrm{CAG}$ and endocrine cell hyperplasia with respect to patients with CAG alone [12] and in patients with type 1 gNEN with respect to patients with CAG alone [16]. However, only Vannella et al., in a recent comparison between 15 patients with type 1 gNEN and 199 patients with CAG alone, reported $\mathrm{CgA}$ as a risk factor of gNEN in univariate analysis [17]. In this study, the role of $\mathrm{CgA}$ was confirmed in a larger cohort of patients $(81$ gNEN and $126 \mathrm{CAG}$ ) and with univariate and multivariate analyses. In particular, our study found a best cutoff range of $59-61 \mathrm{U} / \mathrm{L}$ with $61.5 \%$ of sensitivity and $68.5 \%$ of specificity to differentiate the presence of type 1 gNENs in patients with CAG. As reported by Modlin et al, CgA increase in several gastroenteropancreatic, cardiovascular, pulmonary, rheumatologic and endocrine conditions, and by gastric acid suppressive therapy [14]. For this reason, the role of $\mathrm{CgA}$ in clinical practice is weak and it is mandatory to make an accurate anamnestic evaluation of the patients in order to exclude other causes of an increasing $\mathrm{CgA}$.

Another important risk factor for type $1 \mathrm{gNEN}$ seems to be intestinal metaplasia of the corpus. It has been shown that the risk of type $1 \mathrm{gNEN}$ in subjects with intestinal metaplasia and CAG was higher than in those having CAG without intestinal metaplasia. Gastric intestinal metaplasia is defined as the replacement of the surface, foveolar and glandular epithelium in the gastric mucosa by the intestinal epithelium with the presence of Paneth cells, goblet cells and absorptive cells [18]. To date, several studies have suggested that atrophic gastritis and intestinal metaplasia are major precursor lesions of gastric cancer (not the neuroendocrine type) [19]. The risk of gastric cancer in subjects with severe fundal atrophic gastritis was 5.76 times greater than in those having little or no fundal atrophic gastritis [20]. In addition, 
the subjects with intestinal metaplasia of the corpus have a higher risk of developing gastric cancer with respect to patients without intestinal metaplasia [21, 22].

For the first time, our data suggested a correlation between type $1 \mathrm{gNEN}$ and intestinal metaplasia of the corpus. These data could exemplify the effects of prolonged disease and prolonged hyperplastic action of gastrin on gastric ECL-cells [2]. In fact, it is well known that, in patients with CAG, gastrin exerts trophic effects upon ECLcells which undergo hyperplasia and, in some cases, progression to type 1 gNEN occurs.

In clinical practice, the greater risk of gastric cancer in patients with intestinal metaplasia rather than the risk of a gNEN, suggests a strict screening interval [23].

The correlation between more prolonger hyperplastic action of gastrin on gastric ECL-cells and the risk of a type 1 gNEN seems to be confirmed by another risk factor for type 1 gNEN: the age of the patient. In fact, in this study, it was shown that the risk of gNEN increases with the increased age of the patient. At univariate and multivariate analyses, age greater than 59 years, was a significant risk factor for a gNEN. Our data is in contrast with the previously published by Vannella who did not show any correlation with patient age [17].

Finally, in contrast with Vannella et al., our study found another independent risk factor for gNEN in male gender [17]. In our data $60.4 \%$ of males with CAG and $31.8 \%$ of females had a gNEN. This data should be confirmed with large epidemiological study.

In our study, the absence of an autoimmune pattern (negative of APCAs) and the presence of severe CGA were risk factors for gNEN at univariate analysis but not at multivariate analysis. These data strengthen what had been reported by Vannella who did not show any correlation between gNENs, and grade of atrophy or autoimmune pattern.

Nicolaou A described a high diagnostic accuracy of gastrin level for ECL cell hyperplasia identification in patients with Hashimoto's thyroiditis [24]. Vannella described a significantly higher value in patients with type 1 gNENs if compared to patients with CAG alone. In our study, the median gastrin level was similar in patients with type 1 gNENs and patients with CAG alone. This discordance needs to be investigated in specific prospective studies.

The main limitation of our study was the lack of ECLdysplasia evaluation. In fact, in 2013, Vanoli et al. and in 2011, Vannella described ECL-dysplasia as a strong risk factor of type 1 gNENs $[17,25]$. Another limitation of our study was the retrospective design of the study. On the other hand, this study represents the first evaluation of risk factors of type 1 gNENs in a large cohort of patients with neoplastic disease.
Another limitation of our study was the high specialization in neuroendocrine tumours in participating centres with a consecutive selection bias (large number of CAG with gNETs). For this reason, this is a retrospective case-control study without an epidemiological evaluation. In fact, we had $39,1 \%$ of patients with gNEN while in Vanella L. et al. only $2.4 \%$ of patients with GAC had gNEN [17]. Alexandraki KI et al. in 2014 had reported that $23 \%$ of patients with Hashimoto's thyroiditis and ECL hyperplasia had gNEN [26].

In conclusion, this study showed that high levels of $\mathrm{CgA}$, the presence of intestinal metaplasia, male gender and age $>58$ years represent independent risk factors for type 1 gNENs. These risk factors should be considered when planning the timing for screening. To better evaluate this aspect, prospective studies are necessary.

Acknowledgments All authors approved the final version of the article, including the authorship list.

\section{Compliance with ethical standards}

Conflict of interest Dr. Antongiulio Faggiano has received research grants from NOVARTIS Farma s.p.a. and IPSEN PHARMA. The other authors declare that they have no conflict of interest.

\section{References}

1. M. Rugge, P. Correa, M.F. Dixon, R. Fiocca, T. Hattori, J. Lechago, G. Leandro, A.B. Price, P. Sipponen, E. Solcia, H. Watanabe, R.M. Genta, Gastric mucosal atrophy: interobserver consistency using new criteria for classification and grading. Aliment. Pharmacol. Ther. 16, 1249-1259 (2002)

2. M.D. Burkitt, D.M. Pritchard, Review article: pathogenesis and management of gastric carcinoid tumours. Aliment. Pharmacol. Ther. 24, 1305-1320 (2006)

3. G. Rindi, O. Luinetti, M. Cornaggia, C. Capella, E. Solcia, Three subtypes of gastric argyrophil carcinoid and the gastric neuroendocrine carcinoma: a clinicopathologic study. Gastroenterology 104, 994-1006 (1993)

4. H.L. Waldum, A.K. Sandvik, J.R. Idle, Gastrin is the most important factor in ECL tumorigenesis. Gastroenterology 114, 1113-1115 (1998)

5. K. Borch, B. Ahren, H. Ahlman, S. Falkmer, G. Granerus, L. Grimelius, Gastric carcinoids: biologic behavior and prognosis after differentiated treatment in relation to type. Ann Surg. 242, 64-73 (2005)

6. K. Borch, H. Renvall, G. Liedberg, Gastric endocrine cell hyperplasia and carcinoid tumors in pernicious anemia. Gastroenterology 88, 638-648 (1985)

7. G. Rindi, C. Azzoni, S. La Rosa, C. Klersy, D. Paolotti, S. Rappel, M. Stolte, C. Capella, C. Bordi, E. Solcia, ECL cell tumor and poorly differentiated endocrine carcinoma of the stomach: prognostic evaluation by pathological analysis. Gastroenterology 116, 532-542 (1999)

8. O. Hosokawa, Y. Kaizaki, M. Hattori, K. Douden, H. Hayashi, M. Morishita, K. Ohta, Long-term follow up of patients with 
multiple gastric carcinoids associated with type A gastritis. Gastric Cancer 8, 42-46 (2005)

9. M.F. Dixon, R.M. Genta, J.H. Yardley, P. Correa, Classification and grading of gastritis. The updated Sydney System. International Workshop on the Histopathology of Gastritis, Houston 1994. Am. J. Surg. Pathol. 20, 1161-1181 (1996)

10. F.T. Bosman, F. Carneiro, R.H. Hruban, N.D. Theise. WHO Classification of Tumors of the Digestive System. 4th edn. IARC, Lyon, (2010)

11. G. Rindi, G. Kloppel, H. Alhman, M. Caplin, A. Couvelard, W.W. de Herder, B. Erikssson, A. Falchetti, M. Falconi, P. Komminoth, M. Korner, J.M. Lopes, A.M. McNicol, O. Nilsson, A. Perren, A. Scarpa, J.Y. Scoazec, B. Wiedenmann, TNM staging of foregut (neuro)endocrine tumors: a consensus proposal including a grading system. Virchows Arch. 449, 395-401 (2006)

12. D. Campana, F. Nori, L. Piscitelli, A.M. Morselli-Labate, R. Pezzilli, R. Corinaldesi, P. Tomassetti, Chromogranin A: is it a useful marker of neuroendocrine tumors? J. Clin. Oncol. 25, 1967-1973 (2007)

13. M.C. Zatelli, M. Torta, A. Leon, M.R. Ambrosio, M. Gion, P. Tomassetti, F. De Brau, G. Delle Fave, L. Dogliotti, E.C. degli Uberti, Chromogranin A as a marker of neuroendocrine neoplasia: an Italian Multicenter Study. Endocr. Relat. Cancer 14, 473-482 (2007)

14. I.M. Modlin, B.I. Gustafsson, S.F. Moss, M. Pavel, A.V. Tsolakis, M. Kidd, Chromogranin A--biological function and clinical utility in neuro endocrine tumor disease. Ann. Surg. Oncol. 17, 2427-2443 (2010)

15. K. Borch, M. Stridsberg, P. Burman, J.F. Rehfeld, Basal chromogranin A and gastrin concentrations in circulation correlate to endocrine cell proliferation in type-A gastritis. Scand. J. Gastroenterol. 32, 198-202 (1997)

16. M. Peracchi, C. Gebbia, G. Basilisco, M. Quatrini, C. Tarantino, C. Vescarelli, S. Massironi, D. Conte, : Plasma chromogranin A in patients with autoimmune chronic atrophic gastritis, enterochromaffin-like cell lesions and gastric carcinoids. Eur. J. Endocrinol. 152, 443-448 (2005)

17. L. Vannella, A. Sbrozzi-Vanni, E. Lahner, C. Bordi, E. Pilozzi, V.D. Corleto, J.F. Osborn, G. Delle Fave, B. Annibale, Development of type I gastric carcinoid in patients with chronic atrophic gastritis. Aliment. Pharmacol. Ther. 33, 1361-1369 (2011)
18. A.C. de Vries, E.J. Kuipers, Epidemiology of premalignant gastric lesions: implications for the development of screening and surveillance strategies. Helicobacter 12(Suppl 2), 22-31 (2007)

19. H. Ohata, S. Kitauchi, N. Yoshimura, K. Mugitani, M. Iwane, H. Nakamura, A. Yoshikawa, K. Yanaoka, K. Arii, H. Tamai, Y. Shimizu, T. Takeshita, O. Mohara, M. Ichinose, Progression of chronic atrophic gastritis associated with Helicobacter pylori infection increases risk of gastric cancer. Int. J. Cancer 109, 138-143 (2004)

20. M. Tatsuta, H. Iishi, A. Nakaizumi, S. Okuda, H. Taniguchi, T. Hiyama, H. Tsukuma, A. Oshima, Fundal atrophic gastritis as a risk factor for gastric cancer. Int. J. Cancer 53, 70-74 (1993)

21. S. Shichijo, Y. Hirata, K. Sakitani, S. Yamamoto, T. Serizawa, R. Niikura, H. Watabe, S. Yoshida, A. Yamada, Y. Yamaji, T. Ushiku, M. Fukayama, K. Koike, Distribution of intestinal metaplasia as a predictor of gastric cancer development. J. Gastroenterol. Hepatol 30, 1260-1264 (2015)

22. W.K. Leung, J.J. Sung, Review article: intestinal metaplasia and gastric carcinogenesis. Aliment. Pharmacol. Ther. 16, 1209-1216 (2002)

23. Y.H. Park, N. Kim, Review of atrophic gastritis and intestinal metaplasia as a premalignant lesion of gastric cancer. J. Cancer Prev. 20, 25-40 (2015)

24. A. Nicolaou, D. Thomas, K.I. Alexandraki, S. Sougioultzis, A.V. Tsolakis, G. Kaltsas, Predictive value of gastrin levels for the diagnosis of gastric enterochromaffin-like cell hyperplasia in patients with Hashimoto's thyroiditis. Neuroendocrinology 99, 118-122 (2014)

25. A. Vanoli, S. La Rosa, O. Luinetti, C. Klersy, R. Manca, C. Alvisi, S. Rossi, E. Trespi, A. Zangrandi, F. Sessa, C. Capella, E. Solcia, Histologic changes in type A chronic atrophic gastritis indicating increased risk of neuroendocrine tumor development: the predictive role of dysplastic and severely hyperplastic enterochromaffin-like cell lesions. Hum. Pathol 44, 1827-1837 (2013)

26. K.I. Alexandraki, A. Nikolaou, D. Thomas, V. Syriou, P. Korkolopoulou, S. Sougioultzis, G. Kaltsas, Are patients with autoimmune thyroid disease and autoimmune gastritis at risk of gastric neuroendocrine neoplasms type 1? Clin. Endocrinol. (Oxf.) 80, 685-690 (2014) 